\title{
Innovations in cancer immunotherapy: chimeric antigen receptor T-cell therapy (CAR-T)
}

\author{
Kevin Brown MD, Matthew D. Seftel MD MPH, Kevin A. Hay MD MSc
}

Cite as: CMAJ 2021 August 23;193:E1300-2. doi: 10.1503/cmaj.202907

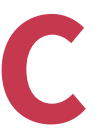

ellular immunotherapy is a novel therapy where the body's own immune cells are harnessed for their anticancer effect. One of the most exciting of these approaches is chimeric antigen receptor T-cell therapy (CAR-T). This therapy is now standard of care for relapsed or refractory B-cell acute lymphoblastic leukemia (B-ALL), the most common malignancy of childhood, and in high-grade B-cell lymphomas, the most common lymphoma in Canada. ${ }^{1}$ CAR-T is undergoing clinical trials in other cancers.

\section{What is CAR-T?}

T cells play a vital role in anticancer surveillance. However, early clinical studies with unmodified T cells showed only modest efficacy. With the advent of genetic engineering techniques, investigators were able to use nonreplicating viral vectors that modify T cells in order to express a "chimeric antigen receptor" (CAR), which provides external specificity for cancer antigens linked to T-cell activation. ${ }^{2}$ A major breakthrough occurred when CAR-T directed against CD19, a B-cell marker, showed a clinical response in most patients, with long-term responses in a small proportion of patients with relapsed or refractory B-ALL and high-grade B-cell lymphoma. This led to US Food and Drug Administration and Health Canada approval for these indications. $^{3}$

\section{How is CAR-T delivered?}

The manufacturing and administration of CAR-T is outlined in Figure 1. Various CAR-T products are available, each with variations in the type of viral vector or manufacturing process. Before CAR-T is administered, patients are given a short course of immunosuppressive chemotherapy to allow CAR-T cells to proliferate after infusion. In Canada, CAR-T is administered at blood and marrow transplant centres that care for patients receiving complex cellular therapy.

\section{Who is eligible for CAR-T?}

The therapy is approved in Canada for patients with B-ALL up to age 25 years, who are either refractory to first-line treatment, have relapsed after allogeneic hematopoietic cell transplant, or

\section{Key points}

- Chimeric antigen receptor T-cell therapy (CAR-T) is a targeted, gene-edited cell therapy for cancer that is approved in Canada for relapsed or refractory high-grade B-cell lymphoma and B-cell acute lymphoblastic leukemia.

- Most patients with relapsed refractory high-grade B-cell lymphoma and B-cell acute lymphoblastic leukemia have progression-free and overall survival rates better than historical controls, and a small proportion are cured.

- Acute toxicities of CAR-T are cytokine release syndrome and immune effector cell-associated neurotoxicity syndrome, and longer-term toxicities include prolonged cytopenias and B-cell aplasia.

- Clinical trials investigating the efficacy of CAR-T in other malignancies, including solid tumours, are underway.

who have had a second or later relapse. It is also approved for adults with relapsed or refractory high-grade B-cell lymphomas who have previously received 2 or more lines of therapy.

\section{What is the evidence so far?}

Table 1 summarizes the data on the effectiveness of currently available CAR-T products. Historically, patients with relapsed or refractory B-ALL had 1-year survival rates of about $30 \%$; patients with relapsed or refractory high-grade B-cell lymphoma have a median overall survival of 6.2 months with standard chemotherapy approaches. ${ }^{5}$

\section{What are the harms of CAR-T?}

In the first month after administration of CAR-T, close clinical monitoring is required for 2 unique toxicities. The first is cytokine release syndrome (CRS), which results from the activation of the immune system and expression of inflammatory cytokines. ${ }^{10}$ Fever is the defining feature, with hypotension and hypoxia characteristic of severe toxicity. In advanced cases, disseminated intravascular coagulation, multi-organ failure and death can occur. ${ }^{10}$ Both corticosteroids and the interleukin-6 (IL-6) receptor antagonist, tocilizumab, can ameliorate CRS manifestations. Intensive care unit support may be required for severe cases. ${ }^{10}$ 


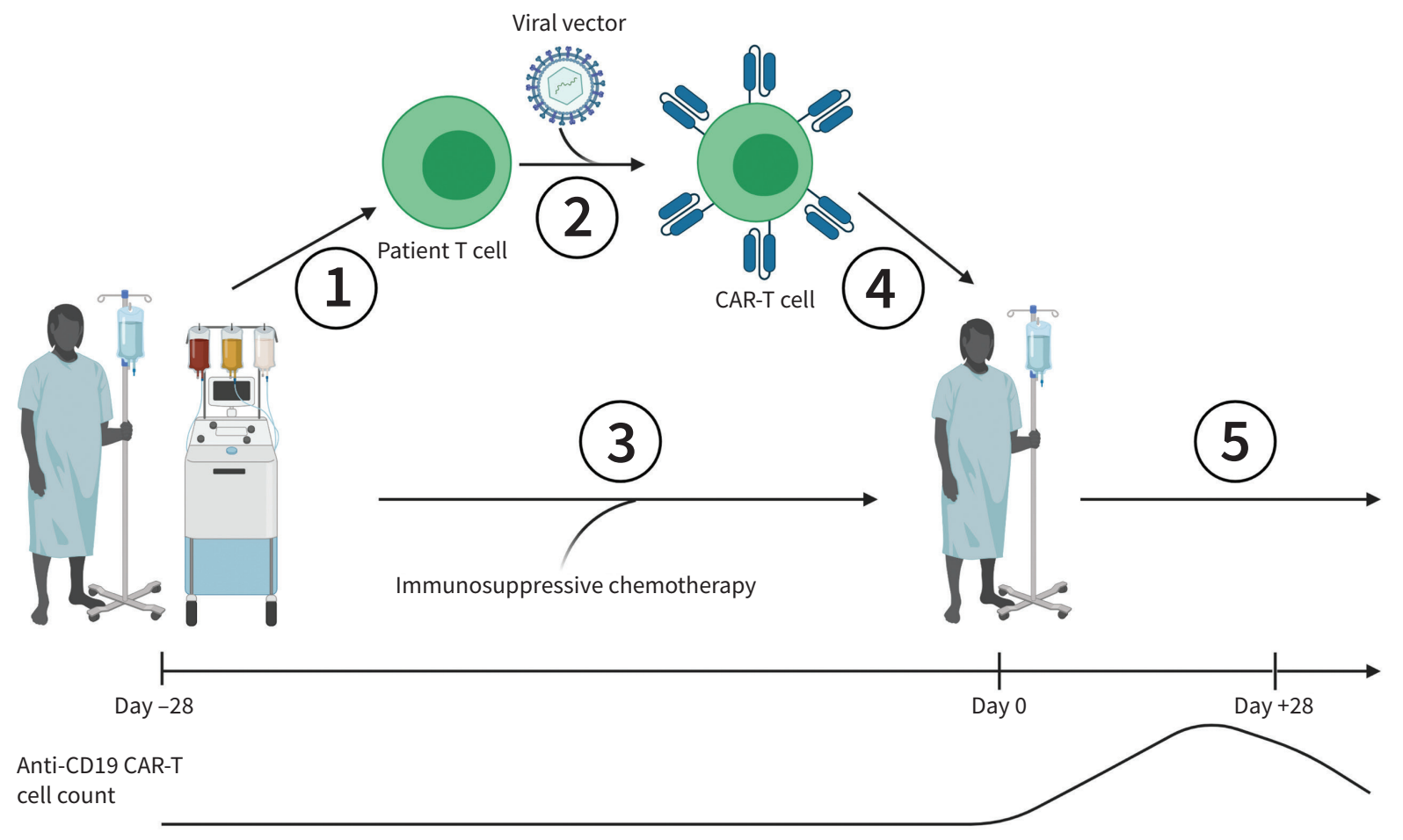

Figure 1: Process of manufacturing and administration of chimeric antigen receptor T-cell therapy (CAR-T). 1. The patient's T cells are collected via apheresis and transported to a manufacturing facility. 2. The patient's T cells are transduced with a viral vector and allowed to proliferate in vitro. 3. The patient is treated with immunosuppressive chemotherapy while the CAR-T cells are manufactured. 4. The CAR-T cells are transported back to the patient's centre and infused. 5. The patient is monitored for cytokine release syndrome (CRS) and immune effector cell-associated neurologic syndrome for up to 28 days and monitored long term for B-cell hypoplasia, hypogammaglobulinemia, cytopenias and opportunistic infections. Manufacturing timeline (not to scale) and patient CAR-T cell count are also shown.

\section{Table 1: Summary of current efficacy data of commercial CAR-T products*}

\begin{tabular}{|c|c|c|c|}
\hline CAR-T product & Disease(s) treated & Antigen target & Survival data \\
\hline Tisagenlecleucel $\dagger^{4,5}$ & $\begin{array}{l}\text { B-ALL and high-grade B-cell } \\
\text { lymphoma }\end{array}$ & CD19 & $\begin{array}{l}\text { - } 76 \% \text { OS at } 1 \text { year in B-ALL } \\
\text { - Median OS of } 8.3 \text { mo in high-grade B-cell lymphoma }\end{array}$ \\
\hline Axicabtagene ciloleucel $\dagger^{6}$ & High-grade B-cell lymphoma & CD19 & $58 \%$ OS at $18 \mathrm{mo}$ \\
\hline Brexucabtagene autoleucel $\dagger^{7}$ & Mantle cell lymphoma & CD19 & $83 \%$ OS at $1 \mathrm{yr}$ \\
\hline Idecabtagene vicleucel $\dagger^{8}$ & Multiple myeloma & BCMA & Median PFS of $11.8 \mathrm{mo}$ \\
\hline Lisocabtagene maraleucel $\ddagger^{9}$ & High-grade B-cell lymphoma & CD19 & Median OS of $18.8 \mathrm{mo}$ \\
\hline
\end{tabular}

The second is immune effector cell-associated neurotoxicity syndrome (ICANS), which manifests as word-finding difficulty, aphasia or confusion. Severe ICANS results in decreased level of consciousness, motor impairment and cerebral edema. ${ }^{10}$ The pathophysiology of ICANS is less well understood than that of CRS. A disturbed blood-brain barrier in the setting of severe systemic inflammation is likely a key process that leads to ICANS. Although CAR-T cells have been found in the cerebrospinal fluid after infusion, it is unclear whether there is direct neurotoxicity as a result of these migrant cells. ${ }^{9}$ Corticosteroids are the primary treatment for ICANS. Although CRS is a complex and potentially life-threatening syndrome that requires management by a number of different specialists, it is usually self-limiting. Although long-term neurologic sequalae from ICANS have been reported, most cases fully resolve.

Cytokine release syndrome often mimics sepsis, and sepsis can co-occur with CRS, so all patients should be investigated and empirically treated with broad-spectrum antibiotic therapy in 
addition to CRS-directed therapy. Immune effector cellassociated neurotoxicity syndrome has a variable presentation, but all patients should undergo urgent investigation for other possible neurologic causes, such as stroke, with neurology consultation recommended. For any patient within the first month of CAR-T, a cellular therapy physician should be consulted.

Other toxicities of CAR-T include prolonged cytopenias and B-cell hypoplasia, the latter associated with hypogammaglobulinemia and risk of opportunistic infections. Loss of immunity to previously administered vaccinations can occur after immunosuppressive chemotherapy. Primary care physicians must be clinically vigilant in order to recognize opportunistic infections and vaccine-preventable infections in CAR-T patients. Revaccination can typically commence 6 months after CAR-T administration when B-cell reconstitution has occurred. CAR-T is being administered during the current COVID-19 pandemic, with patients consequently at risk of severe SARS-CoV-2 infection. In line with national and international consensus statements, vaccination against SARS-CoV-2 infection is recommended for CAR-T recipients. Given that CAR-T cells are engineered using a nonreplicating viral vector, there is no concern for oncogenic transformation.

\section{What can be expected in the future?}

Currently, more than 500 clinical trials of CAR-T are registered and actively recruiting patients with various malignant and nonmalignant conditions, showing the rapid pace of innovation in this field. However, substantial challenges currently limit the wider adoption of this therapy. It is expensive, with the 2018 cost of both tisagenlecleucel (tisa-cel) and axicabtagene ciloleucel (axi-cel) in the United States at US\$373000 when used for highgrade B-cell lymphomas. ${ }^{11}$ This does not include the costs of supportive care and hospital stay. For lymphoma, an analysis by the Canadian Agency for Drugs and Technologies in Health estimated that both tisa-cel and axi-cel at current prices had low probabilities of being cost-effective at willingness-to-pay thresholds of Can\$50000 or Can\$100000 per quality-adjusted life-year gained. ${ }^{12,13}$ In a publicly funded health care system where resource allocation is strained, cost reduction will be necessary in order to meet expanding indications for CAR-T. Across Canada, access to CAR-T remains a challenge, as not all provinces have finalized the complex legal agreements and business plans necessary for implementation and scale-up of the resources needed for CAR-T. As such, products approved by Health Canada are not yet routinely available for many Canadians unless they are referred to other centres, either in Canada or other countries. Novel strategies such as point-of-care manufacturing, in vivo gene delivery to immune cells using nanoparticles (thus circumventing the need for cell manufacturing), and "off the shelf," allogeneic (i.e., using donor T cells) products are in active development and may reduce cost and access challenges by reducing the costs of individual patient engineering costs.

\section{References}

1. Brenner DR, Weir HK, Demers AA, et al. Projected estimates of cancer in Canada in 2020. CMAJ 2020;192:E199-205.

2. Gross G, Waks T, Eshhar Z. Expression of immunoglobulin-T-cell receptor chimeric molecules as functional receptors with antibody-type specificity. Proc Natl Acad Sci U S A 1989;86:10024-8.

3. Brentjens RJ, Latouche JB, Santos E, et al. Eradication of systemic B-cell tumors by genetically targeted human $T$ lymphocytes co-stimulated by CD 80 and interleukin-15. Nat Med 2003;9:279-86.

4. Maude SL, Laetsch TW, Buechner J, et al. Tisagenlecleucel in children and young adults with B-cell lymphoblastic leukemia. N Engl J Med 2018;378:439-48.

5. Schuster SJ, Bishop MR, Tam CS, et al. Tisagenlecleucel in adult relapsed or refractory diffuse large B-cell lymphoma. N Engl J Med 2019;380:45-56.

6. Neelapu SS, Locke FL, Bartlett NL, et al. Axicabtagene ciloleucel CAR T-cell Therapy in refractory large B-Cell lymphoma. N Engl J Med 2017;377:2531-44.

7. Wang M, Munoz J, Goy A, et al. KTE-X19 CAR T-cell therapy in relapsed or refractory mantle-cell lymphoma. N Engl J Med 2020;382:1331-42.

8. Raje N, Berdeja J, Lin Y, et al. Anti-BCMA CAR T-cell therapy bb2121 in relapsed or refractory multiple myeloma. N Engl J Med 2019;380:1726-37.

9. Abramson JS, Palomba ML, Gordon LI, et al. Lisocabtagene maraleucel for patients with relapsed or refractory large B-cell lymphomas (TRANSCEND NHL 001): a multicentre seamless design study. Lancet 2020;396:839-52.

10. Neelapu SS. Managing the toxicities of CAR T-cell therapy. Hematol Oncol 2019;37(Suppl 1):48-52.

11. Lin JK, Muffly LS, Spinner MA, et al. Cost effectiveness of chimeric antigen receptor T-cell therapy in multiply relapsed or refractory adult large B-cell lymphoma. J Clin Oncol 2019;37:2105-19.

12. CADTH optimal use report: Tisagenlecleucel for acute lymphoblastic leukemia and diffuse large B-cell lymphoma: recommendations. Ottawa: CADTH; 2019. Available: https://www.cadth.ca/sites/default/files/pdf/car-t/op0538 -recommendations-report-jan2019.pdf (accessed 2021 Feb. 10).

13. CADTH optimal use report: Axicabtagene ciloleucel for large B-cell lymphoma: recommendations. Ottawa: CADTH; 2019. Available: https://www.cadth.ca/ sites/default/files/pdf/car-t/ct0002-axi-cel-recommendations-redacted report. pdf (accessed 2021 Feb. 10).

Competing interests: Matthew Seftel reports receiving consulting fees from the Canadian Agency for Drugs and Technologies in Health, Novartis and Kite/Gilead. Kevin Hay has served as an ad hoc consultant on advisory boards for Kite/Gilead, Celgene/BMS, and Novartis. No other competing interests were declared.

This article has been peer reviewed.

Affiliations: Leukemia and Bone Marrow Transplant Program of British Columbia (Hay); Division of Hematology, Department of Medicine (Seftel, Hay), University of British Columbia; Canadian Blood Services (Seftel); Terry Fox Laboratory (Hay), British Columbia Cancer Research Institute, Vancouver, BC; Department of Internal Medicine (Brown), Max Rady College of Medicine, University of Manitoba, Winnipeg, Man.

Contributors: All of the authors contributed to the conception and design of the work, drafted the manuscript, revised it critically for important intellectual content, gave final approval of the version to be published and agreed to be accountable for all aspects of the work.

Content licence: This is an Open Access article distributed in accordance with the terms of the Creative Commons Attribution (CC BY-NCND 4.0) licence, which permits use, distribution and reproduction in any medium, provided that the original publication is properly cited, the use is noncommercial (i.e., research or educational use), and no modifications or adaptations are made. See: https://creativecommons.org/ licenses/by-nc-nd/4.0/

Correspondence to: Kevin Hay, khay@bccrc.ca 